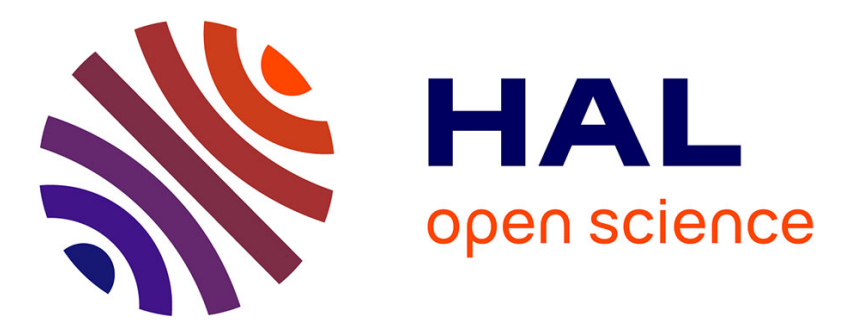

\title{
Solving the Homogeneous Isotropic Linear Elastodynamics Equations Using Potentials and Finite Elements. The Case of the Rigid Boundary Condition
}

Aliénor Burel, Sébastien Imperiale, Patrick Joly

\section{- To cite this version:}

Aliénor Burel, Sébastien Imperiale, Patrick Joly. Solving the Homogeneous Isotropic Linear Elastodynamics Equations Using Potentials and Finite Elements. The Case of the Rigid Boundary Condition. Numerical Analysis and Applications, 2012, 5 (2), pp.136-143. 10.1134/S1995423912020061 . hal00717160

\author{
HAL Id: hal-00717160 \\ https://hal.inria.fr/hal-00717160
}

Submitted on 12 Jul 2012

HAL is a multi-disciplinary open access archive for the deposit and dissemination of scientific research documents, whether they are published or not. The documents may come from teaching and research institutions in France or abroad, or from public or private research centers.
L'archive ouverte pluridisciplinaire HAL, est destinée au dépôt et à la diffusion de documents scientifiques de niveau recherche, publiés ou non, émanant des établissements d'enseignement et de recherche français ou étrangers, des laboratoires publics ou privés. 


\title{
Solving the Homogeneous Isotropic Linear Elastodynamics Equations Using Potentials and Finite Elements. The Case of the Rigid Boundary Condition
}

\author{
Aliénor Burel ${ }^{1,2 \star}$, Sébastien Impériale ${ }^{1 \star \star}$ and Patrick Joly ${ }^{1 \star \star \star}$ \\ 1 POEMS, UMR 7231, CNRS-ENSTA-INRIA, INRIA, Domaine de Voluceau, 78153 Le Chesnay Cedex, France, \\ 2 Université Paris-Sud XI, Laboratoire d'Analyse Numérique, 91405 Orsay cedex, France.
}

\begin{abstract}
In this article, elastic wave propagation in a homogeneous isotropic elastic medium with rigid boundary is considered. A method based on the decoupling of pressure and shear waves via the use of scalar potentials, is proposed. This method is adapted to a finite elements discretization, which is discussed. A stable, energy preserving numerical scheme is presented, as well as $2 \mathrm{D}$ numerical results.
\end{abstract}

\section{Introduction}

Decomposing the displacement into potentials is a well-known tool in elastodynamics, and it expresses the decoupling of the pressure wave and the shear wave inside a homogeneous isotropic media. Although this tool is classically used when searching for analytic solutions [1], it does not seem to have been exploited for numerical computation using finite elements for instance. However, this is a priori attractive since, contrary to a displacement field approach for instance [5], it allows to decouple the approximation of $\mathrm{P}$ and $\mathrm{S}$ waves and to adapt the discretization process (mesh size, order of elements) to the dynamics of each type of wave, which is a priori particularly interesting when S-waves propagate much more slowly than Pwaves (soft materials such as rubber). The main difficulty is to cope with the coupling of the different types of waves (the so-called conversion of modes) which occurs, due to wave reflections and transmissions, at interfaces between homogeneous media or at physical boundaries. The simplest situation where this phenomenon appears is the propagation of elastic waves in a homogeneous domain with clamped boundary. This is the model problem that we address in this paper.

\section{Potentials Formulation of the Elastodynamics Problem}

In what follows, we consider a two-dimensional space, and we will use the following notations for differential operators: $\left(x=\left(x_{1}, x_{2}\right)\right.$ will denote the space variable, $\mathbf{u}=\left(u_{1}, u_{2}\right)$ a vector field and $u$ a scalar function)

$$
\begin{cases}\nabla u=\left(\begin{array}{c}
\partial_{x_{1}} u \\
\partial_{x_{2}} u
\end{array}\right), & \overrightarrow{\operatorname{curl}} u=\left(\begin{array}{c}
\partial_{x_{2}} u \\
-\partial_{x_{1}} u
\end{array}\right), \\
\operatorname{curl} \mathbf{u}=\partial_{x_{1}} u_{2}-\partial_{x_{2}} u_{1}, & \operatorname{div} \mathbf{u}=\partial_{x_{1}} u_{1}+\partial_{x_{2}} u_{2},\end{cases}
$$

and we recall:

$$
-\Delta u=-\operatorname{div}(\nabla u)=\operatorname{curl}(\overrightarrow{\operatorname{curl}} u), \quad \operatorname{div}(\overrightarrow{\operatorname{curl}} u)=0, \quad \operatorname{curl}(\nabla u)=0 .
$$

In the particular case of an isotropic homogeneous elastic media into a domain $\Omega \subset \mathbb{R}^{2}$, the linear elastodynamics equations that characterize the variations of the displacement field $\mathbf{u}(x, t)$ are [1]:

$$
\rho \frac{\partial^{2} \mathbf{u}}{\partial t^{2}}-(\lambda+2 \mu) \nabla(\operatorname{div} \mathbf{u})+\mu \overrightarrow{\operatorname{curl}}(\operatorname{curl} \mathbf{u})=0, \quad x \in \Omega, \quad t>0
$$

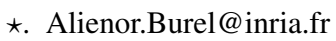

$\star \star$. Sebastien.Imperiale@inria.fr

$\star \star \star$. Patrick.Joly@inria.fr
} 
where $\rho>0$ is the (constant) material density, $\lambda>0$ and $\mu>0$ its Lamé coefficients (also constant). In the following, we want to solve the elastodynamics equations (2) in a domain $\Omega \subset \mathbb{R}^{2}$ with rigid boundary $\Gamma=\partial \Omega$ and we complete (2) with homogeneous Dirichlet boundary conditions:

$$
\mathbf{u}=0, \quad \text { on } \Gamma, \quad t>0
$$

To define a well-posed problem, one must provide initial conditions to (2) and (3):

$$
\mathbf{u}(x, 0)=\mathbf{u}_{0}(x), \quad \partial_{t} \mathbf{u}(x, 0)=\mathbf{u}_{1}(x), \quad x \in \Omega
$$

In the following, we introduce the decomposition of the displacement field with the help of potentials. Although this is classical, we recall this notion for completeness and for fixing notations. From (2), we easily see, using (1), that the divergence and the (scalar) curl of the vector field, respectively $\mathbf{d}=\operatorname{div} \mathbf{u}$ and $\mathbf{r}=$ curl $\mathbf{u}$ verify a scalar wave equation, respectively:

$$
\rho \frac{\partial^{2} \mathbf{d}}{\partial t^{2}}-(\lambda+2 \mu) \Delta \mathbf{d}=0, \quad \rho \frac{\partial^{2} \mathbf{r}}{\partial t^{2}}-\mu \Delta \mathbf{r}=0,
$$

where the speeds associated with these equations are the P-wave (pressure wave) speed $V_{P}$ and $\mathrm{S}$-wave (shear wave) speed $V_{S}$, namely:

$$
V_{P}^{2}=\frac{\lambda+2 \mu}{\rho}, \quad V_{S}^{2}=\frac{\mu}{\rho} .
$$

This observation suggests to seek the displacement field in terms of a decomposition into potentials using the Hodge-Helmoltz decomposition (see [6] for more details):

$$
\mathbf{u}=\nabla \varphi_{P}+\overrightarrow{\operatorname{curl}} \varphi_{S}
$$

where the $\mathrm{P}$ and $\mathrm{S}$ wave potentials $\varphi_{P}$ and $\varphi_{S}$ verify a scalar wave equation:

$$
\rho \frac{\partial^{2} \varphi_{P}}{\partial t^{2}}-(\lambda+2 \mu) \Delta \varphi_{P}=0, \quad \rho \frac{\partial^{2} \varphi_{S}}{\partial t^{2}}-\mu \Delta \varphi_{S}=0, \quad x \in \Omega, \quad t>0 .
$$

Indeed, using again (1), it is easy to verify that if $\varphi_{P}$ and $\varphi_{S}$ are solutions of (6), then the vector field $\mathbf{u}$ given by (5) is solution of (2). We have:

$$
\rho \frac{\partial^{2} \mathbf{u}}{\partial t^{2}}=\nabla\left(\rho \frac{\partial^{2} \varphi_{P}}{\partial t^{2}}\right)+\overrightarrow{\operatorname{curl}}\left(\rho \frac{\partial^{2} \varphi_{S}}{\partial t^{2}}\right)
$$

Using (6) and (1), we get:

$$
\rho \frac{\partial^{2} \mathbf{u}}{\partial t^{2}}=(\lambda+2 \mu) \nabla\left(\operatorname{div}\left(\nabla \varphi_{P}\right)\right)+\mu \overrightarrow{\operatorname{curl}}\left(\operatorname{curl}\left(\overrightarrow{\operatorname{curl}} \varphi_{S}\right)\right) .
$$

This equality is not modified if we write, thanks to (1) once again:

$$
\rho \frac{\partial^{2} \mathbf{u}}{\partial t^{2}}=(\lambda+2 \mu) \nabla\left(\operatorname{div}\left(\nabla \varphi_{P}+\overrightarrow{\operatorname{curl}} \varphi_{S}\right)\right)+\mu \overrightarrow{\operatorname{curl}}\left(\operatorname{curl}\left(\nabla \varphi_{P}+\overrightarrow{\operatorname{curl}} \varphi_{S}\right)\right)
$$

and we obtain (2) by using (5) again. Therefore, we can see that the equations that characterize the P and $\mathrm{S}$ waves, that is to say $\varphi_{P}$ and $\varphi_{S}$, are totally decoupled in $\Omega$, but coupled on the border through the boundary conditions, in our case for a rigid boundary:

$$
\nabla \varphi_{P}+\overrightarrow{\operatorname{curl}} \varphi_{S}=0, \quad \text { on } \Gamma
$$

We will use this last equation in a more natural way in what follows, by projecting it on normal and tangent vectors. To achieve this, we introduce along $\Gamma$ the unit outgoing vector normal to $\Gamma, n=\left(n_{1}, n_{2}\right)$ and a curvilinear abscissa $\tau \in \mathbb{R}$, oriented such as any smooth enough scalar function $\varphi$ defined on $\Omega$ satisfies:

$$
\frac{\partial \phi}{\partial \tau}=\nabla \phi \times n, \quad \text { on } \Gamma
$$


where, for all $(x, y) \in \mathbb{R}^{2} \times \mathbb{R}^{2}$, we denote: $x \times y:=x_{1} y_{2}-x_{2} y_{1}$. If we use the notation:

$$
\frac{\partial \phi}{\partial n}:=\nabla \phi \cdot n, \quad \text { on } \Gamma \quad\left(x \cdot y:=x_{1} y_{1}+x_{2} y_{2}\right),
$$

we easily check that:

$$
\overrightarrow{\operatorname{curl}} \varphi \cdot n=-\frac{\partial \varphi}{\partial \tau}, \quad \text { and } \quad \overrightarrow{\operatorname{curl}} \varphi \times n=\frac{\partial \varphi}{\partial n}, \quad \text { on } \Gamma
$$

Therefore, (7) is equivalent to the scalar equations:

$$
\frac{\partial \varphi_{P}}{\partial n}-\frac{\partial \varphi_{S}}{\partial \tau}=0, \quad \text { and } \quad \frac{\partial \varphi_{S}}{\partial n}+\frac{\partial \varphi_{P}}{\partial \tau}=0, \quad \text { on } \Gamma
$$

The potentials problem will be to find two scalar functions:

$$
\varphi_{P}(x, t): \Omega \times \mathbb{R}^{+} \longrightarrow \mathbb{R}, \quad \varphi_{S}(x, t): \Omega \times \mathbb{R}^{+} \longrightarrow \mathbb{R}
$$

satisfying the 'inside' equation (6), the boundary conditions (9) and the initial conditions (11):

$$
\begin{cases}\varphi_{P}(x, 0)=\varphi_{p, 0}(x), \quad \varphi_{S}(x, 0)=\varphi_{S, 0}(x), & x \in \Omega \\ \partial_{t} \varphi_{P}(0, x)=\varphi_{p, 1}(x), \partial_{t} \varphi_{S}(0, x)=\varphi_{S, 1}(x), & x \in \Omega .\end{cases}
$$

We established, at least formally, the link between this problem and the initial problem $(2,3,4)$. We write it as a 'theorem' which we choose to state without mathematical framework, for simplicity :

Proposition 1. If $\varphi_{P}$ and $\varphi_{S}$ are solutions of $(6,9,11)$, then the vector field $\mathbf{u}$ (the displacement field) defined by (5) is solution of $(2,3,4)$ with the following initial conditions:

$$
\mathbf{u}_{0}=\nabla \varphi_{P, 0}+\overrightarrow{\operatorname{curl}} \varphi_{S, 0}, \quad \mathbf{u}_{1}=\nabla \varphi_{P, 1}+\overrightarrow{\operatorname{curl}} \varphi_{S, 1} .
$$

Remark 1. Proposition above has naturally a reciprocal: if we decouple the initial data $\left(\mathbf{u}_{0}, \mathbf{u}_{1}\right)$ into potentials such as (12), then we can decompose the solution $\mathbf{u}$ of $(2,3,4)$ into potentials such as $(5)$, where $\varphi_{P}$ and $\varphi_{S}$ are solutions of $(6,9,11)$.

\section{Variational and Energetic Approach of the Potentials Problem}

\subsection{Energy Conservation}

The formal equivalence between $(2,3,4)$ and the potentials problem $(6,9,11)$ 'shows' that this last one is well-posed. Nonetheless, it is useful to have a direct proof of the well-posedness to solve it numerically. Such a proof will be a consequence of an energy equality result, as expected. However, there is a small trick, as we will see, insofar as the conservative energy is not the sum (nor any convex combination) of the energies naturally associated with the two wave equations (6), that is to say:

$$
E \neq \frac{1}{2} \int_{\Omega}\left(\rho\left|\frac{\partial \varphi_{P}}{\partial t}\right|^{2}+(\lambda+2 \mu)\left|\nabla \varphi_{P}\right|^{2}\right)+\frac{1}{2} \int_{\Omega}\left(\rho\left|\frac{\partial \varphi_{S}}{\partial t}\right|^{2}+\mu\left|\nabla \varphi_{S}\right|^{2}\right)
$$

which would be a conservative energy if, for instance, each potential was vanishing on the boundary (and in that case, the two potentials would be totally decoupled). In fact, as we can expect, the right energy which we obtain from such a combination will differ only from a boundary term. In order to see that, we will start from the energy identity obtained from the equations (6) after dividing them by $\lambda+2 \mu$ and $\mu$ respectively, multiplying by $\partial_{t} \varphi_{P}$ and $\partial_{t} \varphi_{S}$ respectively, and integrating the result over $\Omega$. We obtain:

$$
\left\{\begin{array}{l}
\frac{d}{d t}\left[\frac{1}{2} \int_{\Omega}\left(\frac{1}{V_{P}^{2}}\left|\frac{\partial \varphi_{P}}{\partial t}\right|^{2}+\left|\nabla \varphi_{P}\right|^{2}\right)\right]=\int_{\Gamma} \frac{\partial \varphi_{P}}{\partial n} \frac{\partial \varphi_{P}}{\partial t} \\
\frac{d}{d t}\left[\frac{1}{2} \int_{\Omega}\left(\frac{1}{V_{S}^{2}}\left|\frac{\partial \varphi_{S}}{\partial t}\right|^{2}+\left|\nabla \varphi_{S}\right|^{2}\right)\right]=\int_{\Gamma} \frac{\partial \varphi_{S}}{\partial n} \frac{\partial \varphi_{S}}{\partial t} .
\end{array}\right.
$$


Then, we use the boundary conditions (9), replacing the normal derivatives with tangential derivatives, as they appear in (9). We sum these two equalities to obtain:

$$
\frac{1}{2} \frac{d}{d t}\left[\int_{\Omega}\left(\frac{1}{V_{P}^{2}}\left|\frac{\partial \varphi_{P}}{\partial t}\right|^{2}+\left|\nabla \varphi_{P}\right|^{2}+\frac{1}{V_{S}^{2}}\left|\frac{\partial \varphi_{S}}{\partial t}\right|^{2}+\left|\nabla \varphi_{S}\right|^{2}\right)\right]=\int_{\Gamma}\left(\frac{\partial \varphi_{S}}{\partial \tau} \frac{\partial \varphi_{P}}{\partial t}-\frac{\partial \varphi_{P}}{\partial \tau} \frac{\partial \varphi_{S}}{\partial t}\right) .
$$

Here, it is useful to introduce some notations. We will consider:

$$
\Phi:=\left(\varphi_{P}, \varphi_{S}\right) \in H^{1}(\Omega)^{2}, \quad \Psi:=\left(\psi_{P}, \psi_{S}\right) \in H^{1}(\Omega)^{2},
$$

and introduce the bilinear form:

$$
b(\Phi, \Psi):=\int_{\Gamma}\left(\frac{\partial \varphi_{S}}{\partial \tau} \psi_{P}-\frac{\partial \varphi_{P}}{\partial \tau} \psi_{S}\right), \quad \forall(\Phi, \Psi) \in H^{1}(\Omega)^{2} \times H^{1}(\Omega)^{2},
$$

so that the second side of (13) is:

$$
b\left(\Phi(t), \frac{d \Phi}{d t}(t)\right) .
$$

We observe that, with some integrations by parts along the border (details are left to the reader), the bilinear form $b(\Phi, \Psi)$ is symmetric, and that leads to:

$$
b\left(\Phi(t), \frac{d \Phi}{d t}(t)\right)=\frac{d}{d t}\left[\frac{1}{2} b(\Phi(t), \Phi(t))\right],
$$

so that (13) can be rewritten:

$$
\frac{d}{d t} E(t)=0
$$

where the function $E(t)$-which is an energy as we will see later, cf. lemma 1-is given by:

$E(t):=\frac{1}{2}\left[\int_{\Omega}\left(\frac{1}{V_{P}^{2}}\left|\frac{\partial \varphi_{P}}{\partial t}\right|^{2}+\left|\nabla \varphi_{P}\right|^{2}\right)+\int_{\Omega}\left(\frac{1}{V_{S}^{2}}\left|\frac{\partial \varphi_{S}}{\partial t}\right|^{2}+\left|\nabla \varphi_{S}\right|^{2}\right)-\int_{\Gamma}\left(\frac{\partial \varphi_{S}}{\partial \tau} \varphi_{P}-\frac{\partial \varphi_{P}}{\partial \tau} \varphi_{S}\right)\right]$.

To ensure that $E(t)$ is an 'energy', particularly a positive function, we can rely on the following lemma:

Lemma 1. We have, for all $\left(\varphi_{P}, \varphi_{S}\right)$ in $H^{1}(\Omega)^{2}$,

$$
\int_{\Omega}\left(\left|\nabla \varphi_{P}\right|^{2}+\left|\nabla \varphi_{S}\right|^{2}\right)-\int_{\Gamma}\left(\frac{\partial \varphi_{S}}{\partial \tau} \varphi_{P}-\frac{\partial \varphi_{P}}{\partial \tau} \varphi_{S}\right)=\int_{\Omega}\left|\nabla \varphi_{P}+\overrightarrow{\operatorname{curl}} \varphi_{S}\right|^{2}
$$

Proof. We restrict ourselves to smooth enough $\varphi_{P}$ and $\varphi_{S}$, and we conclude by density and continuity. We first develop the right term of (17) to obtain:

$$
\int_{\Omega}\left|\nabla \varphi_{P}+\overrightarrow{\operatorname{curl}} \varphi_{S}\right|^{2}=\int_{\Omega}\left(\left|\nabla \varphi_{P}\right|^{2}+\left|\nabla \varphi_{S}\right|^{2}\right)+2 \int_{\Omega} \nabla \varphi_{P} \cdot \overrightarrow{\operatorname{curl}} \varphi_{S}
$$

where we used $\left|\nabla \varphi_{S}\right|=\left|\overrightarrow{\operatorname{curl}} \varphi_{S}\right|$. Then, we use the Green's formula:

$$
\int_{\Omega} \nabla \varphi_{P} \cdot \overrightarrow{\operatorname{curl}} \varphi_{S}=-\int_{\Omega} \varphi_{P} \operatorname{div}\left(\overrightarrow{\operatorname{curl}} \varphi_{S}\right)+\int_{\Gamma} \overrightarrow{\operatorname{curl}} \varphi_{S} \cdot n \varphi_{P},
$$

thus, using (1) and (8),

$$
2 \int_{\Omega} \nabla \varphi_{P} \cdot \overrightarrow{\operatorname{curl}} \varphi_{S}=-2 \int_{\Gamma} \frac{\partial \varphi_{S}}{\partial \tau} \varphi_{P}=-\int_{\Gamma}\left(\frac{\partial \varphi_{S}}{\partial \tau} \varphi_{P}-\frac{\partial \varphi_{P}}{\partial \tau} \varphi_{S}\right) .
$$

We obtain the second inequality by integrating by parts along the boundary $\Gamma$, and this proof is achieved. A corollary of this lemma is that the function $E(t)$ is a positive energy, and another expression of $E$ is:

$$
E(t)=\frac{1}{2} \int_{\Omega}\left(\frac{1}{V_{P}^{2}}\left|\frac{\partial \varphi_{P}}{\partial t}\right|^{2}+\frac{1}{V_{S}^{2}}\left|\frac{\partial \varphi_{S}}{\partial t}\right|^{2}\right)+\frac{1}{2} \int_{\Omega}\left|\nabla \varphi_{P}+\overrightarrow{\operatorname{curl}} \varphi_{S}\right|^{2} .
$$




\subsection{Variational Formulation of the Problem}

In this section, we consider the Hilbert spaces:

$$
V:=V_{P} \times V_{S}, \quad V_{P}=H^{1}(\Omega)^{2}, \quad V_{P}=H^{1}(\Omega)^{2},
$$

and the bilinear forms:

$$
m(\Phi, \Psi):=m_{P}\left(\varphi_{P}, \psi_{P}\right)+m_{S}\left(\varphi_{S}, \psi_{S}\right), \quad a(\Phi, \Psi):=a_{P}\left(\varphi_{P}, \psi_{P}\right)+a_{S}\left(\varphi_{S}, \psi_{S}\right),
$$

where we denoted:

$$
m_{Q}\left(\varphi_{Q}, \psi_{Q}\right):=\frac{1}{V_{Q}^{2}} \int_{\Omega} \varphi_{Q} \psi_{Q}, \quad a_{Q}\left(\varphi_{Q}, \psi_{Q}\right):=\int_{\Omega} \nabla \varphi_{Q} \cdot \nabla \psi_{Q}, \quad Q \in\{P, S\} .
$$

Then, it is a simple exercise to see that the weak space formulation of $(6,9,11)$ can be written as:

$$
\left\{\begin{array}{l}
\text { Find } \Phi: \mathbb{R}^{+} \rightarrow V \text { satisfying (11) such that: } \\
\frac{d^{2}}{d t^{2}} m(\Phi(t), \Psi)+a(\Phi(t), \Psi)-b(\Phi(t), \Psi)=0, \quad \forall \Psi \in V .
\end{array}\right.
$$

In what follows, we consider an abstract problem of the framework (22). We can denote that:

- Theoretically, the important properties will be:

$$
\left\{\begin{array}{l}
\text { The bilinear forms } m(\cdot, \cdot), a(\cdot, \cdot) \text { and } b(\cdot, \cdot) \text { are continuous and symmetric on } V, \\
\text { The bilinear form } m(\cdot, \cdot), \text { is positive definite, } \\
\text { The bilinear form } a(\cdot, \cdot)-b(\cdot, \cdot) \text { is positive. }
\end{array}\right.
$$

In our case, these properties are satisfied with (19), (20), (21) and (15), thanks to lemma 1.

- From a practical point of view it is important that $V=V_{P} \times V_{s}$ and that we have a decomposition of $m(\cdot, \cdot)$ and $a(\cdot, \cdot)$ as (20), which means that the unknowns $\varphi_{P}$ and $\varphi_{S}$ are just linked by $b(\cdot, \cdot)$. Regarding the present application, the fact that $b(\cdot, \cdot)$ is an integral on the boundary (15) will have practical consequences: the numerical treatment of the coupling will be less expensive.

\section{An Energy preserving Discretization Scheme}

\subsection{Construction and stability analysis}

We first discretize in space by a Galerkin method, introducing a family of finite dimension subspaces $V_{h}$ of $V$, where $h$ is an (abstract) approximation parameter. One can write the semi-discrete problem as follows (in order to simplify, we pass over the handling of the initial data):

$$
\left\{\begin{array}{l}
\text { Find } \Phi_{h}: \mathbb{R}^{+} \rightarrow V_{h} \text { such that: } \\
\frac{d^{2}}{d t^{2}} m\left(\Phi_{h}(t), \Psi_{h}\right)+a\left(\Phi_{h}(t), \Psi_{h}\right)-b\left(\Phi_{h}(t), \Psi_{h}\right)=0, \quad \forall \Psi_{h} \in V
\end{array}\right.
$$

which modulo some abusive but obvious notation that we omit to precise, is equivalent to a finite dimension differential system:

$$
\mathbf{M}_{h} \frac{d^{2} \Phi_{h}}{d t^{2}}+\mathbf{A}_{h} \Phi_{h}-\mathbf{B}_{h} \Phi_{h}=0,
$$

where, using (23), $\mathbf{M}_{h}, \mathbf{A}_{h}$ and $\mathbf{B}_{h}$ are symmetric matrices, $\mathbf{M}_{h}$ is positive definite and $\mathbf{A}_{h}-\mathbf{B}_{h}$ is positive. Then we discretize in time with a leap-frog scheme, and we allow ourselves to treat differently 
(we will explain why later) the terms $a\left(\Phi_{h}(t), \Psi_{h}\right)$, treated explicitely and $b\left(\Phi_{h}(t), \Psi_{h}\right)$, treated implicitely with a $\theta$-scheme. The numerical scheme can be written $(\theta \in] 0,1[)$ in its variational form:

$$
\left\{\begin{array}{l}
\text { Find } \Phi_{h}^{n} \in V_{h}, \quad(n=0,1,2, \cdots) \text { such that, for all } \Psi_{h} \text { in } V: \\
m\left(\frac{\Phi_{h}^{n+1}-2 \Phi_{h}^{n}+\Phi_{h}^{n-1}}{\Delta t^{2}}, \Psi_{h}\right)+a\left(\Phi_{h}^{n}, \Psi_{h}\right)-b\left(\theta \Phi_{h}^{n+1}+(1-2 \theta) \Phi_{h}^{n}+\theta \Phi_{h}^{n-1}, \Psi_{h}\right)=0
\end{array}\right.
$$

or in its algebrical form:

$$
\mathbf{M}_{h} \frac{\Phi_{h}^{n+1}-2 \Phi_{h}^{n}+\Phi_{h}^{n-1}}{\Delta t^{2}}+\mathbf{A}_{h} \Phi_{h}^{n}-\mathbf{B}_{h}\left(\theta \Phi_{h}^{n+1}+(1-2 \theta) \Phi_{h}^{n}+\theta \Phi_{h}^{n-1}\right)=0 .
$$

The interest of this framework of schemes is that if $\theta \geq 1 / 4$, its stability condition is independent of the bilinear form $b(\cdot, \cdot)$. We prove it in the following:

Theorem 1. When $\theta \geq 1 / 4$, the scheme (26) is stable as long as:

$$
\theta \Delta t^{2}\left\|\mathcal{A}_{h}\right\| \leq 1, \quad \text { where } \quad\left\|\mathcal{A}_{h}\right\|:=\sup _{\Psi_{h} \in V_{h} \backslash\{0\}} \frac{a\left(\Psi_{h}, \Psi_{h}\right)}{m\left(\Psi_{h}, \Psi_{h}\right)} .
$$

Proof. We use an energy method. We first point out that:

$$
\theta \Phi_{h}^{n+1}+(1-2 \theta) \Phi_{h}^{n}+\theta \Phi_{h}^{n-1}=\Phi_{h}^{n}+\theta \Delta t^{2} \frac{\Phi_{h}^{n+1}-2 \Phi_{h}^{n}+\Phi_{h}^{n-1}}{\Delta t^{2}},
$$

to rewrite (26) as:

$$
m_{\Delta t}^{*}\left(\frac{\Phi_{h}^{n+1}-2 \Phi_{h}^{n}+\Phi_{h}^{n-1}}{\Delta t^{2}}, \Psi_{h}\right)+a^{*}\left(\theta \Phi_{h}^{n+1}+(1-2 \theta) \Phi_{h}^{n}+\theta \Phi_{h}^{n-1}, \Psi_{h}\right)=0,
$$

where we denote:

$$
m_{\Delta t}^{*}(\Phi, \Psi):=m(\Phi, \Psi)-\theta \Delta t^{2} a(\Phi, \Psi), \quad a^{*}(\Phi, \Psi):=a(\Phi, \Psi)-b(\Phi, \Psi) .
$$

Then, we can see that:

$$
\theta \Phi_{h}^{n+1}+(1-2 \theta) \Phi_{h}^{n}+\theta \Phi_{h}^{n-1}=\frac{\Phi_{h}^{n+1}+2 \Phi_{h}^{n}+\Phi_{h}^{n-1}}{4}+\left(\theta-\frac{1}{4}\right) \Delta t^{2} \frac{\Phi_{h}^{n+1}-2 \Phi_{h}^{n}+\Phi_{h}^{n-1}}{\Delta t^{2}}
$$

to rewrite (26) as:

$$
\begin{aligned}
m_{\Delta t}^{*}\left(\frac{\Phi_{h}^{n+1}-2 \Phi_{h}^{n}+\Phi_{h}^{n-1}}{\Delta t^{2}}, \Psi_{h}\right)+\left(\theta-\frac{1}{4}\right) & \Delta t^{2} a^{*}\left(\frac{\Phi_{h}^{n+1}-2 \Phi_{h}^{n}+\Phi_{h}^{n-1}}{\Delta t^{2}}, \Psi_{h}\right) \\
& +a^{*}\left(\frac{\Phi_{h}^{n+1}+2 \Phi_{h}^{n}+\Phi_{h}^{n-1}}{4}, \Psi_{h}\right)=0 .
\end{aligned}
$$

If we choose $\Psi_{h}=\frac{\Phi_{h}^{n+1}-\Phi_{h}^{n-1}}{2 \Delta t}$, we obtain a result of energy conservation:

$$
\frac{1}{\Delta t}\left(E_{h}^{n+\frac{1}{2}}-E_{h}^{n-\frac{1}{2}}\right)=0
$$

where we denote:

$$
\begin{aligned}
E_{h}^{n+\frac{1}{2}}:=\frac{1}{2} m_{\Delta t}^{*}\left(\frac{\Phi_{h}^{n+1}-\Phi_{h}^{n}}{\Delta t}, \frac{\Phi_{h}^{n+1}-\Phi_{h}^{n}}{\Delta t}\right) & +\frac{1}{2}\left(\theta-\frac{1}{4}\right) \Delta t^{2} a^{*}\left(\frac{\Phi_{h}^{n+1}-\Phi_{h}^{n}}{\Delta t}, \frac{\Phi_{h}^{n+1}-\Phi_{h}^{n}}{\Delta t}\right) \\
& +\frac{1}{\Delta t} a^{*}\left(\frac{\Phi_{h}^{n+1}+\Phi_{h}^{n}}{2}, \frac{\Phi_{h}^{n+1}+\Phi_{h}^{n}}{2}\right)
\end{aligned}
$$

Classically, the stability of the scheme is a consequence of the positivity of this energy. To conclude, one need to keep in mind that, by hypothesis, the bilinear form $a^{*}(\cdot, \cdot)$ is positive and (28) is nothing but the time step condition on $\Delta t$ needed for the positivity of $m_{\Delta t}^{*}(\cdot, \cdot)[4]$. 
Remark 2. The stability condition (28) taken strictly implies the inversibility of the matrix $M_{h}-\theta \Delta t^{2} B_{h}$, the matrix we have to inverse on each time step of the algorithm.

Remark 3. We notice that considering (28), we obtain the classical stability condition by taking $\theta=1 / 4$, it is the condition that we obtain by solving the two equations independently. In the following, we always take $\theta=1 / 4$.

\subsection{Computational aspects}

We use high-order Lagrangian finite elements on quadrilateral grids (also called spectral elements as in [2]) with a priori different meshes and orders for each potential adapted to each wave speed (a similar idea has also been developed in a nearby context in [3] for fluid-structure interaction). We also use a mass lumping technique [2] in order to have a mass matrix $\mathbf{M}_{h}$ seen in equation (25) diagonal. As a consequence, the computation is purely explicit, except for the degrees of freedom located on the boundary, which are coupled through the matrix $\mathbf{B}_{h}$. Moreover, $\mathbf{B}_{h}$ has the structure of a 1D finite element matrix so that the additional cost due to the computation of the boundary unknowns is marginal.

\section{Numerical Results}

In our experiments, the domain of computation is a 2D annulus. We have chosen a S-wave 'point source' term (a localized in space right hand side in equation (6), that varies in time as a Ricker pulse). Speaking of the meshes, we took the same quadrangular meshes for the P-wave and the S-wave, but different orders: 3 for the P-wave and 6 for the $\mathrm{S}$-wave, taking into account the shorter wavelength for $\mathrm{S}$ waves: $V_{P}$ is taken about 3 times bigger than $V_{S}$.

In figure (1), we displayed snapshots of numerical solutions (to be seen column per column) corresponding, from top to bottom, to instants 1.6, 2, 2.4, 2.8, and 5. The first three columns correspond to calculations made with our new method. On the first two columns, we represent the modulus of the displacement for the $\mathrm{P}$ and $\mathrm{S}$ waves respectively, that is to say $\nabla \varphi_{P}$ and $\overrightarrow{\operatorname{curl}} \varphi_{S}$, whereas we represent in the third column, the modulus of the total displacement field $\nabla \varphi_{P}+\overrightarrow{\operatorname{curl}} \varphi_{S}$. In the last column, we represent the same quantity for anther numerical solution computed with a finite element approach for the classical displacement formulation. We observe a very good agreement between the results in columns 3 and 4 .

\section{Conclusion}

In this paper, we have proposed a quite satisfactory solution, from both theoretical and practical points of view, for the model problem of section 1. However, this remains insufficient with respect to applications. For this type of method to be convincing, we should be able to treat transmission problems and free boundary conditions. We expect that if we get a good solution for the free boundary condition, then we shall de able to deal with transmission conditions. Unfortunately, this problem appears to be much more challenging than the one treated in this paper: the first attempts that we have done so far, although they seem to work for the time harmonic problem, meet severe instability problems with the time dependent case. Solving this major difficulty appears to be the bottleneck for future developments in the direction of this paper.

\section{References}

1. J Achenbach. Wave Propagation in Elastic Solids. North Holland, January 1975.

2. G Cohen. Higher-order numerical methods for transient wave equations. Springer-Verlag, Berlin, 2001.

3. J Diaz and P Joly. Robust high order non-conforming finite element formulation for time domain fluid-structure interaction. Journal of Computational Acoustics. An IMACS Journal, 13(3):403-431, 2005. 
4. P Joly. Variational Methods for Time Dependant Wave Propagation. In M Ainsworth, P Davies, D Duncan, P Martin, and B Rynne, editors, Topics in computational wave propagation : Direct and Inverse Problems. Computational Methods in Wave Propagation, pages 201-264. Springer Verlag, October 2003.

5. P Joly. Finite element methods with continuous displacement. pages 267-329. Chapman \& Hall/CRC, Boca Raton, FL, 2008.

6. P Monk. Finite element methods for Maxwell's equations. Oxford science publications, 2003. 


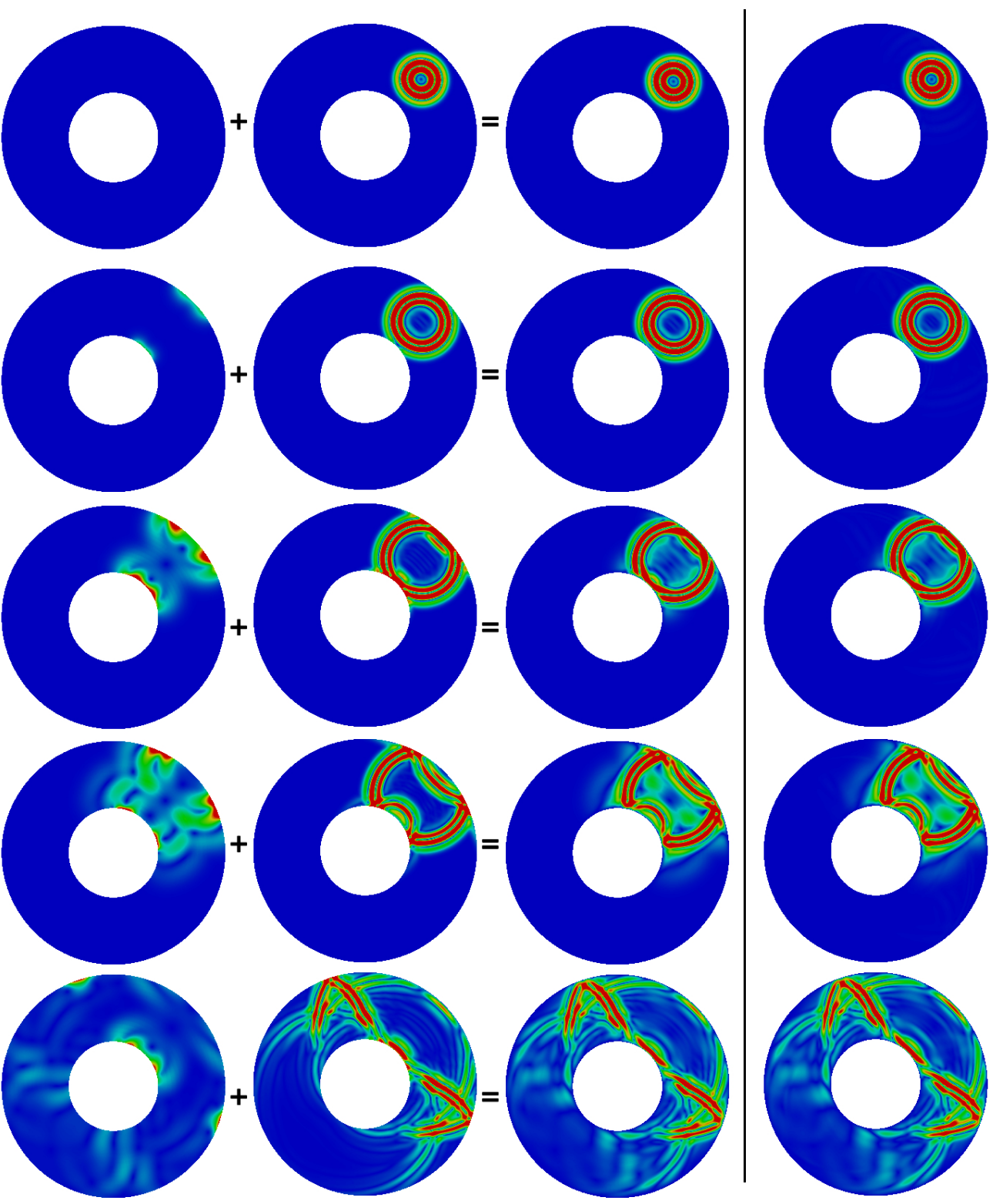

Fig. 1. Propagation of an S-wave in a disk. From left to right: modulus of the P-wave, the S-wave, the sum of the two waves and $\mathbf{u}$ solution of the elastodynamic equation. 